\title{
ON A PROBLEM OF ROTA
}

\author{
BY E. RODNEY CANFIELD
}

Communicated by J. R. Goldman, August 8, 1977

Let $S(n, k)$ denote the Stirling numbers of the second kind, and let $K_{n}$ be such that $S\left(n, K_{n}\right) \geqslant S(n, k)$ for all $k$. Rota's problem [3] is to prove or disprove the following:

For all $n$, the largest possible incomparable collection of partitions of an $n$ set contains $S\left(n, K_{n}\right)$ partitions.

An "incomparable collection" of partitions is one in which no partition in the collection is a refinement of some other partition in the collection.

Definition. Let $S(n, k)$ denote the collection of all partitions of an $n$ set into $k$ nonempty blocks. If $C \subseteq S(n, k)$, define $\operatorname{Span}(C)$ by

$\operatorname{Span}(\mathcal{C})=\left\{\pi \in S(n, k+1): \pi\right.$ is a refinement of some $\left.\pi^{\prime} \in \mathcal{C}\right\}$.

THEOREM. For all sufficiently large $n$, there is a collection $C \subseteq S(n, j)$ such that

(i) $j+1=K_{n}$,

(ii) $|\operatorname{Span}(\mathrm{C})|<|\mathrm{C}|$, where || denotes cardinality.

Consequently, $(S(n, j+1)-\operatorname{Span}(C)) \cup C$ is an incomparable collection with more than $S\left(n, K_{n}\right)$ partitions.

REMARKS. C consists of all $\pi \in S(n, j)$ having exactly $l$ blocks of size $\leqslant M$ and exactly $j-l$ blocks of size $>M$ and $\leqslant 2 M$, where $l$ and $M$ are appropriately defined.

The proof of the Theorem requires [2] to estimate $|C|$ and $|\operatorname{Span}(C)|$; and also requires [1] to know the approximate value of $K_{n}$.

\section{REFERENCES}

1. E. Rodney Canfield, On the location of the maximum Stirling number(s) of the second kind, 1977 (preprint).

2. - Application of the Berry-Esseen inequality to combinatorial estimates, 1977 (preprint).

3. G.C. Rota, Research problem 2-1, J. Combinatorial Theory 2 (1967), 104.

DEPARTMENT OF STATISTICS AND COMPUTER SCIENCE, UNIVERSITY OF GEORGIA, ATHENS, GEORGIA 30602

AMS (MOS) subject classifications (1970). Primary 05A17, 05 A05; Secondary 05C35, 06A10, 05 C 99. 